Voix et Images

volxetimages

\title{
L'irradiation poétique. Entretien avec Fernand Ouellette
}

\section{Noël Audet}

Volume 5, numéro 3, printemps 1980

Fernand Ouellette

URI : https://id.erudit.org/iderudit/200224ar

DOI : https://doi.org/10.7202/200224ar

Aller au sommaire du numéro

Éditeur(s)

Les Presses de l'Université du Québec

ISSN

0318-9201 (imprimé)

1705-933X (numérique)

Découvrir la revue

Citer ce document

Audet, N. (1980). L'irradiation poétique. Entretien avec Fernand Ouellette. Voix et Images, 5(3), 435-469. https://doi.org/10.7202/200224ar d'utilisation que vous pouvez consulter en ligne.

https://apropos.erudit.org/fr/usagers/politique-dutilisation/ 


\section{L'irradiation poétique Entretien avec Fernand Ouellette}

\section{La poésie est ailleurs}

N.A. Fernand Ouellette, vous êtes né à Montréal. Or il y a une image du poète qui nous pousse plus souvent à le faire naître à la campagne ou à Natashquan par exemple... J'aimerais connaître quel rapport vous établissez entre ce lieu de votre naissance et votre œuvre, surtout poétique. Est-ce que vous y voyez des liens directs?

F.O. Sans doute que si j'étais né à la campagne, j'aurais écrit une poésie différente. Je pense à des poètes comme Gaston Miron, Jean-Guy Pilon qui sont nés à la campagne et qui ont fait une poésie autre que la mienne. Je ne suis pas sûr que le fait de naître à Montréal ait eu une grande influence, sinon sur ma sensibilité qui s'est nourrie à la fois de la ville et de la campagne.

N.A. Il y a chez vous effectivement beaucoup d'images d'eau, plus d'images marines que d'évocations de l'asphalte ou du béton.

F.O. En effet, la présence de la ville se réduit à quelques poèmes de Radiographies et de Séquences de l'aile. Ce qui est important, pour moi, ce sont les images qui se situent au-delà même de l'asphalte et des poteaux de téléphone. Ce qu'on appelle le "réel " immédiat ne me paraît certainement pas primordial.

N.A. Vous n'avez jamais été tenté de mythifier la ville moderne?

F.O. Non! Sans doute que j'ai été marqué par le fleuve. Je vivais alors en face des îles de Boucherville, bien que je sois né rue Bennett, dans le quartier de Maisonneuve. J'avais neuf ans, lorsque ma famille s'est établie près de Montréal-Est, devant le fleuve.

N.A. Votre enfance s'est donc déroulée plutôt dans un milieu réaliste ou assez dur. Alors je voudrais savoir comment et pourquoi Fernand Ouellette s'en est échappé. Est-ce qu'il s'agit d'un rejet de ce milieu?

F.O. Pas consciemment. En fait le problème de la poésie n'est pas celui du 
roman. Je suis arrivé au roman beaucoup plus tard. Alors ces problèmes ne se poseront pas pour moi. Mon père n'était pas un ouvrier. Il était un artisan, un ébéniste, c'est tout autre chose. Enfant, je travaillais souvent avec lui dans son atelier. Mon père n'était pas aliéné dans la mesure où il fabriquait des objets, de beaux objets. Je n'ai donc pas eu ce sentiment d'appartenance à une classe sociale, comme si j'avais été un assisté social. Mais comme mon père travaillait le plus souvent pour des riches, je savais que nous étions pauvres, sans être dans la misère.

N.A. On peut effectivement distinguer plusieurs formes de pauvreté : celles du paysan, de l'artisan, de l'ouvrier. Dans les deux premiers cas joue une dimension d'autonomie plus grande, de libre disposition de son temps.

F.O. Mon père travaillait à la fois pour les autres, et pour lui. Il était employé de Henry Morgan \& Co. Et moi-même, à partir de l'âge de treize ans, j'y ai travaillé comme assistant-journalier. C'est le caractère anglophone du milieu qui m'a marqué, comme cela avait dú marquer mon père, et mon grand-père maternel qui y avait travaillé quelque temps.

N.A. Alors quand vous parlez du problème de la langue au Québec, vous savez de quoi vous parlez?

F.O. C'est une chose que j'ai dans le sang, car mon père et moi avons vécu ces problèmes. Je suis donc très sensibilisé à cette question de bilinguisme, et ce n'est pas du tout un problème urbain, mais une question socio-politique et culturelle.

N.A. J'enchaînerai avec une question que je me suis posée et que vousmême vous vous posez dans Les Actes retrouvés. Vous dites que la nature ne serait pour vous qu'un réservoir d'images, est-ce à dire que vous en faites une réalité seconde, par rapport à quoi? Qu'est-ce qui vient d'abord, si ce n'est pas la nature?

F.O. C'est la première réalité sur le plan des sensations. Je puise là où je suis, c'est-à-dire que je me nourris constamment de ce que je reçois à travers tous mes sens. Pour moi c'est primordial la médiation par les sens, c'est fondamental en poésie. Je ne crois pas aux poésies abstraites, aux poésies cérébrales. Je crois aux poésies (malgré ce qu'on a pu dire de moi) qui sont constamment enracinées dans le réel et dans la nature.

N.A. C'est ce que je croyais, mais ce passage m'avait un peu étonné, parce que justement la nature est partout présente dans vos poèmes.

F.O. C'est une nature au sens très large. C'est le réel plus que la nature. Disons «le réel » plutôt que la " réalité " pour parler en termes concrets et non pas abstraits.

N.A. Vous n'êtes tout de même pas d'accord avec Baudelaire - ce poète urbain - quand il disait que la nature est un champ de légumes. II préférait l'artifice, la construction humaine à la nature. 
F.O. S'il ne s'agissait que de cette «nature»-là, je dirais le réel et non pas la nature. Ce serait plus exact.

N.A. Si l'on passait maintenant à un autre aspect de votre poésie. Quels sont les poètes qui vous ont le plus stimulé ou le plus fécondé, qu'ils soient étrangers ou québécois.

F.O. Sur le plan québécois, le premier a vraiment été Alain Grandbois. J'ai eu avec Saint-Denys Garneau un rapport douloureux. Je le rejetais. Mais avec le recul, je pense qu'il est un poète supérieur à Grandbois.

N.A. Parce qu'il y avait une grande parenté sans doute avec Saint-Denys Garneau.

F.O. Oui, une parenté spirituelle. A cette époque-là, et c'est peut-être normal à vingt ans, j'avais tendance à me tourner vers les antipodes de ce que j'étais, pour m'en nourrir.

N.A. Qui était l'antipode?

F.O. Grandbois était vraiment l'antipode pour moi, parce que c'était un homme qui avait voyagé en Orient, en Europe, qui m'ouvrait le monde, quoi! Tandis qu'avec Saint-Denys Garneau, j'avais l'impression de...

N.A. De demeurer dans nos problèmes québécois...

F.O. De caler en moi-même. Il était donc plus urgent d'aller du côté de Grandbois pour m'ouvrir et respirer.

N.A. Une petite parenthése: j'ai pu vérifier, auprès d'étudiants français, que Grandbois est reçu comme un poète très ordinaire, alors que SaintDenys Garneau passe la rampe et séduit.

F.O. Je pense qu'il a plus d'originalité d'écriture, qu'il est un plus grand poète. Plus profond et plus fécond, plus près d'une certaine modernitè dénudée, silencieuse.

N.A. Apparemment, c'est la rhètorique d'Alain Grandbois qui pose problème.

F.O. Enfin, je l'apprécie encore, mais si je suis plus sévère, c'est qu'aujourd'hui je n'ai plus le même rapport avec lui.

N.A. Et vous n'êtes pas remonté jusqu'à des poètes antérieurs? Comme Nelligan?

F.O. J'ai lu Nelligan mais cela ne m'a jamais marqué. La véritable influence d'écriture ce serait Pierre Jean Jouve. II reste le poète qui m'a le plus enrichi. Je devrais dire par la médiation de Pierre Emmanuel, à ce moment-là, car j'ai d'abord connu Emmanuel et ensuite Jouve. Je ne sens pourtant aucune relation poétique avec Emmanuel, aucun rapport avec son type d'écriture. Mais c'est quand même lui qui m'a donné le choc, principalement le choc de l'image. 
N.A. Pierre Emmanuel? II y a tout de même une certaine parenté dans le regard, dans la vision du monde, non?

F.O. Je ne sais pas... De moins en moins... Disons que je ne sens pas d'affinité réelle...

N.A. D'autre part, vous avez traversé très rapidement le surréalisme.

F.O. Oui! En fait je suis venu aux surréalistes, beaucoup plus tard, par Pierre Emmanuel en réalité. Avant de connaître Breton, j'ai connu Pierre Emmanuel, ce qui fait que mon rapport avec le surréalisme n'a pas été très important. Quand j'ai lu Breton, Eluard, je l'ai fait au moment où j'avais déjà eu une influence, qui était très forte, soit celle de Jouve.

N.A. Et René Char?

F.O. J'ai connu René Char personnellement, mais il ne m'a jamais marqué. Sa poésie se situe à la limite du concept et de la pensée, et je suis personnellement porté vers une poésie davantage sensuelle, sensorielle. C'est-à-dire que Jouve m'apparaît beaucoup plus charnel que Char.

N.A. Oui, mais il y a aussi la vie quotidienne dans René Char et ce n'est peutêtre pas tout à fait votre style.

F.O. Ce n'est pas mon style, bien sûr. S'il y avait aujourd'hui des influences de Char au Québec, ce serait indirectement par des disciples tel que Andrè du Bouchet. Mais chez les poètes de ma génération, je ne connais aucune véritable influence d'écriture. Cette poésie "présocratique" n'est pas la mienne du tout.

N.A. Pour revenir à Jouve, qu'est-ce que vous y avez puisé exactement?

F.O. Ce que j'y ai puisé revient à ceci : l'enracinement, et le régard sur la femme, sur le corps. Je vais donner l'exemple concret d'un vers qui comprend trois qualités fondamentales pour moi: le son, le sens et aussi l'irradiation presque lumineuse du son-sens!

\section{Les glaieuls sur elle la plus belle se balancent il fait beau sur sa pierre à mourir de ciel bleu.}

Des sens y participent: glaïeul, fleur précise et non la fleur; sur elle: sur une femme. Se balancent: on perçoit le mouvement. II fait beau sur sa pierre à mourir de ciel bleu: depuis la pierre tombale, quelle lumière dans cette image, qui fait vibrer tout le vers; par les sonorités labiales, quelle radiation musicale! Non seulement ces vers sont beaux, ont un sens, indissociable de leur forme, mais j'y perçois surtout une vibration lumineuse qui caractérise pour moi la grande poésie.

N.A. Oui, c'est du Mallarmé en plus sensuel...

F.O. En plus sensuel, et avec un sens apparemment moins ouvert que celui de Mallarmé. Jouve est plus saisissable et moins hermétique. Et pourtant il dérive de Mallarmé, mais heureusement aussi de Baudelaire. A mon avis, il y a eu une réaction très saine contre Mallarmé, réaction 
qui a été vécue par plusieurs poètes du $X X^{\ominus}$ siècle, comme Apollinaire, Saint-John Perse, Jouve, etc.

N.A. Vous me voyez sourire un peu, parce que j'allais vous demander: il y a de jeunes poètes québécois qui sont très mallarméens...

F.O. Ils sont pour le moins un peu en retard. Sur le plan de l'écriture, la réaction a été faite depuis longtemps. D'autres poètes sont venus après Mallarmé, comme je le disais.

N.A. On ne peut pas avoir plusieurs Mallarmé?

F.O. On en a eu un et cela aboutissait à une impasse.

N.A. Les critiques sont en train de déterrer Mallarmé et d'en faire le plus grand poète de la fin du XIX ${ }^{e}$ siècle, ce qui est peut-être vrai, mais it reste qu'on n'a sans doute pas à refaire du Mallarmé aujourd'hui.

F.O. Je ne pense pas. C'était une époque où l'on pouvait encore rêver le livre unique, presque absolu. Mais cela présente moins d'intérêt aujourd'hui.

N.A. J'évoquais son écriture à la fois ouverte...

F.O. ... précieuse aussi...

N.A. ... plus philosophique, c'est-à-dire chargée de sémantique et en même temps...

F.O. ... de culture, oui...

N.A. ... très travaillée...

F.O. ... très ciselée, très précieuse et c'est peut-être ce qui me rebute le plus aujourd'hui. Je ne dis pas qu'il ne faut pas passer par là, mais la réaction a été saine en tous cas, et principalement celle de Jouve qui à mon sens incarne sa poésie dans sa vie, dans son propre drame, dans ses rapports avec la nature, dans ses rapports avec la femme, c'est en fin de compte beaucoup plus important pour moi.

\section{L'écriture poétique}

N.A. Je vous poserais une question plus difficile en vous demandant d'où vient chez vous, s'il est possible de le définir, la pulsion qui fait naître le poème. S'agit-il avant tout d'un rythme, d'une image, d'une idée, d'une sensation, d'un mouvement encore plus vague?

F.O. Je dirais peut-être que généralement, c'est double: à la fois une sensation et une image, c'est-à-dire une première image.

N.A. Et vous êtes assez conscient quand vous écrivez, pour identifier ce mouvement? 
F.O. Maintenant, après, oui, bien sûr. Je sais par exemple que la plupart des poèmes que j'ai écrits depuis 1960 ont été écrits dans cette rue-ci, à partir de ce que j'y voyais.

N.A. De sensations?

F.O. Oui, à partir de ce que je voyais dans mon jardin ici, un simple jeu de lumière sur les feuilles pouvait déclencher un poème. Une sorte d'étincellement de lumière sur les feuilles. J'ai fait un poème à partir de cette seule sensation. Et combien d'autres à partir de cet arbre-ci devant la maison.

N.A. Mais qui ne parlent pas nécessairement de ça.

F.O. Non, pas du tout.

N.A. Bon, c'est ce que je voulais vous voir préciser.

F.O. C'est un point de départ, je ne sais pas où cela m'emmène. Il y a l'arbre qui est générateur de beaucoup d'images, chez moi. II y a aussi le souvenir de certains lieux. C'est souvent une image concrète, une perception, une sensation très précise qui déclenchent le poème... ou bien c'est une image, sans médiation qui est donnée comme ça, qui vient en lisant, qui vient en pensant à diverses choses... Tout d'un coup l'image apparaît et le poème s'ensuit.

N.A. Après cette première pulsion, cette première impression, qu'est-ce qui se passe?

F.O. Si la tension est forte, tout de suite ça m'oblige à écrire quelque chose, je pars de cette image, et un premier vers vient. Ensuite ça s'enchaine. Je me souviens de peu de cas où j'ai commencé un poème que je n'ai pu terminer.

N.A. Alors là, vous n'êtes pas comme Saint-Denys Garneau, "un poème a mijoté tout le jour...".

F.O. C'est-à-dire que le poème est venu, et j'ai travaillé avec une grande concentration tout de suite sur le texte, de façon à ne pas laisser baisser cette tension.

N.A. Mais est-ce que cette tension se développe ensuite selon une ligne logique ou encore une ligne émotive ou sensorielle?

F.O. Selon toutes ces forces à la fois. C'est totalisant un poème. Je ne sais pas le texte qui est en train de s'écrire. Je n'essaie pas de dire une chose. Un certain nombre d'éléments se cristallisent dans une tension totalisante. C'est une totalité qui se cherche, le poème. Elle se propose comme un absolu. Mais je ne peux pas le savoir au moment où j'écris. Probablement que mes meilleurs poèmes sont ceux qui m'ont le plus échappé, qui m'ont entraîné dans l'écriture, comme malgré moi. Je pense au long poème "Le Périple " publié dans Poésie. Comme ce texte 
me paraissait étranger! Mais souvent ce sont les poèmes les plus déroutants qui sont les plus féconds.

N.A. IIs naissent comme tout faits, ils vous traversent.

F.O. Ils nous traversent. On ne sait pas pourquoi on dit telle chose, pourquoi on écrit ça de telle façon. Ma notion d'errance, au fond, est liée à cette incapacité de savoir ce que j'écris ou ce que je dis.

N.A. Est-ce que ce n'est pas au fond l'inconscient qui écrit à ce moment-là ?

F.O. L'inconscient, le désir sont pour moi dialectiques. C'est une constellation de la mémoire, de l'inconscient, indissociables, et du désir qui polarise tout cela. Le désir ne sait pas ce qu'il veut, il totalise mais ignore où il va nous entraîner. A coup sûr il va quelque part. Prenons l'exemple d'une image dans mon second roman. Mon personnage dit à peu près ceci : Je ne sais pas ce que je suis en train de peindre mais ma main s'arrête, mes doigts ne veulent plus continuer comme s'ils savaient, eux, que le tableau est fini. Ma conscience n'en sait rien, mais les doigts le savent. Le poème également nous échappe. A un moment donné il est terminé. Je peux revenir ensuite pendant deux ou trois ans sur le texte pour le revoir, supprimer dix vers de trop.

N.A. Vous retravaillez beaucoup le poème?

F.O. Je retravaille constamment jusqu'à ce qu'il soit publié.

N.A. En élaguant, plutôt qu'en ajoutant?

F.O. En élaguant toujours. Je ne me souviens pas de beaucoup de cas où j'ai rajouté des choses. Si j'ai rajouté, c'est que j'ai refait un vers à ce moment-là, mais en général je condense.

N.A. Alors vous travaillez en densifiant?

F.O. Oui, toujours.

N.A: On le sent d'ailleurs à la lecture.

F.O. C'est peut-être pour ça qu'il y a beaucoup d'ellipses. La forme est ramassée. Ça semble advenir dans une sorte d'irruption où il n'y aurait ni commencement ni fin. André Brochu a bien remarqué ce phénomène. Quand je commence par une conjonction, par exemple, je fonctionne comme si le discours n'avait jamais été interrompu. Qu'il n'y avait pas eu d'interruption dans mon cheminement poétique.

N.A. Phénomène qui est effectivement très présent chez vous. Les nombreux "car", "or" qui attaquent un poème ou une strophe.

F.O. C'est un travail du poème par la contraction, par la densité, par le mouvement dialectique. Cela appartient sans doute à une conception de la poésie qui est liée à beaucoup d'images d'ascension et de chute, de ruptures continuelles. 
N.A. Est-ce la raison pour laquelle on vous aurait pris pour un poète angélique?

F.O. Comment savoir?

N.A. Ou si c'est plutôt à cause de la signification générale?

F.O. Je pense que mes premiers poèmes disaient peut-être au fond la même chose que j'essaie de dire aujourd'hui, sauf qu'ils le disaient à travers beaucoup moins d'expérience concrète du monde et sur un plan plus abstrait, désincarné. J'ai un vocabulaire qui tend à l'essentiel. Disons que je tends aux mots-souches, aux mots-mères, aux mots fondamentaux.

N.A. Aux nœuds symboliques?

F.O. Je ne suis pas porté à travailler avec les mots du deuxième degré quand je peux m'en tirer autrement. Je m'en tiens à l'essence des choses, mais rendue par la médiation des sens. Je dirai beaucoup plus volontiers l'arbre que l'érable. Je vais vers ce qui est plus englobant, vers les motsmères. La langue française se prête assez facilement à mon jeu. C'est un peu dans sa nature. Peut-être que dans Ces anges de sang, j'étais trop désincarné parce que mon regard n'était pas suffisamment nourri par le monde. Mais à partir du Soleil sous la mort, et même avant, avec Séquences de l'aile, tout s'est concrétisé.

N.A. II y a certainement une évolution très nette, j'y reviendrai à propos du dernier recueil. Mais dans vos poèmes, il y a toujours une tension qui frappe le lecteur, c'est-à-dire une tension entre deux pôles contraires aussi bien au niveau thématique que formel, et je pense que vous-même êtes très conscient de cette dialectique. D'ailleurs le mot "dialectique" revient très souvent sous votre plume, même dans votre roman. Vous avez écrit dans Les Actes retrouvés: "Il ne m'est donc possible de poursuivre ma marche qu'en assumant les deux pôles" - alors je voudrais vous demander de préciser un peu la nature de cette tension. Est-elle d'abord psychologique, c'est-à-dire angoisse en face de la mort en même temps que désir de vie? Enfin est-ce que cette même tension serait devenue politique récemment?

F.O. C'est ma façon d'avoir un rapport avec le monde et les hommes, c'est un rapport qui est tendu et dialectique. II s'exprime sur tous les plans: psychologique, sensoriel, philosophique, si on veut, sociologique... $\mathrm{Ce}$ serait une façon à travers la poésie de dépasser le tragique...

N.A. De dépasser le tragique?

F.O. De surmonter le tragique. Le poème, c'est l'instant où j'ai l'illusion de surmonter le tragique et, inévitablement, il y a rechute, après.

N.A. Oui bien sûr. Comme le disait Rimbaud, c'est la fusion des contraires.

F.O. C'est par cette fusion des contraires que j'ai l'impression de pouvoir 
surmonter le tragique, le temps d'un éclair. C'est la concentration a-temporelle du texte poétique qui me donne cette impression, cette joie. C'est pourquoi la poésie doit être intense, sans quoi elle n'existe tout simplement pas. Surmonter le temps, c'est aussi surmonter ce qui lui appartient : la mort et le tragique. Je crois que ce n'est pas tout à fait une illusion, car il y a une tension de tout l'être dans le poème. En somme, dans l'instant poétique, il y a une sorte de résolution des contraires qui me donne l'éclat d'une percée tragique. C'est à ce sujet-là que je me suis opposé à Kenneth White, lors de la dernière Rencontre des écrivains. Je lui disais que l'instant poétique est une flèche qui perce le tragique, mais que inévitablement la flèche retombe. Kenneth White tente de faire l'économie du tragique. II s'imagine qu'on accède à un espace blanc hors de la condition humaine...

N.A. L'écriture serait cette espèce de réconciliation de tout son être et de tout son être avec le monde aussi, alors je ne vois pas comment on peut sentir la mort à ce moment-là, parce que c'est justement l'expression optimale de la vie.

F.O. Oui, parce que si la conscience de la mort était plus forte, on ne pourrait pas faire de poèmes. Car à ce moment-là, c'est la vie qui l'emparte, et le désir. Mais dans un mouvement dialectique, en sachant très bien que la mort est constamment présente. Il faut se dépêtrer avec la mort, avec le quotidien, avec le monde. C'est pour ça que je ne crois qu'à la poésie qui n'est pas coupée du monde ni de l'histoire. Je pense qu'un poète écrit dans un temps donné et il subit les ondes et les chocs de ce temps, de ce monde-là constamment.

N.A. Vous parlez beaucoup de la mort dans votre œuvre. Est-ce une obsession ou plutôt un sentiment réfléchi, philosophique, ou presque une sensation?

F.O. Cela a commencé d'abord par être une sensation, parce que j'ai failli me noyer à l'âge de neuf ou dix ans. J'ai réussi à rattraper le quai à temps. Mais j'ai eu l'impression, l'instant d'un éclair, que c'était la mort déjà. Plus tard j'ai vu la mort de près, deux fois. Quant à l'expérience de la mort des autres, j'entends celle des gens avec qui j'ai des liens profonds comme ma femme, mon père ou ma mère, mes enfants, mes amis, pour le moment, je ne l'ai pas encore éprouvée.

N.A. Donc c'est vraiment quelque chose qui vient pratiquement de l'enfance et qui se rapproche d'une sensation.

F.O. Oui!

N.A. A cause aussi de votre sensibilité sans doute. Parce que la plupart des gens ont fait face à la mort un jour ou l'autre, mais ils ne s'en sont même pas rendu compte...

F.O. Peut-être. Cheż moi c'est en fait la conscience de l'éphémère, de la vulnérabilité de tout. Cela est certainement lié aussi à la dimension de 
l'au-delà, du religieux bien sûr. Je ne lis pas la mort de la même façon que celui qui ne croit pas à un au-delà. Je pense d'ailleurs que savoir qu'il y a un au-delà est plus angoissant.

N.A. Comment est-ce possible?

F.O. Enfin cela semble contradictoire, mais si on sait que c'est la fin, quelle angoisse peut-il y avoir? C'est la fin, on est un animal.

N.A. Un animal qui aimerait bien se survivre.

F.O. Oui qui aimerait bien se survivre mais en même temps qui est conscient de sa fin, qu'il n'y a rien d'autre qui se passe après.

N.A. Mais c'est encore plus tragique, non?

F.O. Je ne sais pas. C'est le néant, l'absence de conscience. N'est-il pas plus difficile, angoissant d'assumer une forme d'existence inimaginable? Tu vois, c'est tellement douloureux de vivre notre temps, de supporter l'humanité, que la certitude du néant après la mort est peut-être une forme de libération. Mais qu'est-ce que peut bien être cette durée, cette éternité, cette survie, cette résurrection dont on parle? En fait, quelle que soit I'hypothèse que l'on envisage, l'une est peut-être aussi angoissante que l'autre. Croire donne sans doute plus d'espérance, ce n'est pas négligeable.

N.A. D'accord, maintenant, je comprends mieux. C'est le pulsionnel, le désir qui refuse de faire le saut.

F.O. Sans doute le pulsionnel, comme tu dis, le corps, l'imagination. Avoir la conviction que nous retrouverons les êtres aimés, ça permet peut-être de se préparer à faire le saut, mais si on en croit l'expérience du Christ sur la croix, ce n'est pas du tout sûr.

\section{De quel engagement?}

N.A. Vous appartenez, Fernand Ouellette, au mouvement de l'Hexagone, enfin vous avez publié aux éditions de l'Hexagone. Et quant à cette génération de poètes qui ont voulu se donner une identité nationale, comment vous situez-vous? Par exemple, Gaston Miron qui prend en charge un vocabulaire particulier, qui retraverse le folklore pour se donner des racines québécoises et finalement s'identifier à un «nous " collectif qui est assez bien situé dans I'histoire du Québec. Et vous, par rapport à cette volonté, qu'est-ce qui se passe dans vos poèmes ? Vous écrivez dans le Journal Dénoué, "En 1955 (...) j'ètais totalement étranger aux graves interrogations qui commençaient à mettre en question notre communauté ». Mais il s'est passé beaucoup de choses depuis...

F.O. Ma poésie se situe au Québec de toute façon, même si cela peut sembler imperceptible. Je me nourris d'abord du Québec, même si le 
Québec n'est pas l'objet de mes poèmes. Quelquefois il y a des tensions qui collent à notre question nationale, mais très peu. Je pense à certains poèmes du Soleil sous la mort, ou au poème "Octobre 1970 ". Je ne dis pas que je suis très optimiste quand je regarde le Québec, mais peu importe. Ce n'est peut-être qu'une question de sensibilité. Certains camarades me reprochent parfois ma vision sombre de l'histoire.

N.A. Vous n'avez jamais senti le besoin par exemple d'écrire "québécois", quand une certaine mode est passée en poésie? D'aller chercher ce que j'appelle ses racines? Cela ne vous motivait pas au point de départ?

F.O. Cela ne me motivait pas, mais peut-être que le fait, comme tu le disais au début, d'être né à Montréal pose un autre rapport, déjà, avec la terre québécoise. II se fait à travers une ville et à travers l'x étranger " surtout, qui était presque une sorte d'«occupant».

N.A. Mais l'étranger dans votre œuvre, n'est pas du tout senti comme un étranger à rejeter, c'est plutôt un accueil international!

F.O. Bien entendu! C'est d'ailleurs pour aller vers lui que je me suis passionné pour Grandbois. Mais quand je parle de "l'étranger", je pense à celui qui essaie de faire croire que je suis "étranger " dans ma propre ville, mon propre pays; je parle de l'Anglophone. Quand je suis en France, je sais très bien que je ne suis pas français. Je me sens américain de langue française. Je ne remets pas en question mon américanité ou ma québécitude. C'est une évidence première. Mais lorsque je réfléchis à notre histoire collective, là je dois me poser la question de notre survie comme peuple, de notre destin. Et je ne me la pose peut-être pas dans les mêmes termes que Gaston Miron qui est né à Sainte-Agathe, mais je n'en suis pas du tout sûr. Il y a sans doute entre nous des différences de perception, de sensibilité.

N.A. Je comprends très bien, ce n'est pas un reproche que je vous fais.

F.O. Non, c'est comme ça et j'allais dire heureusement parce que si je regarde des poètes de ma génération, par exemple Paul-Marie Lapointe, Gaston Miron, c'est heureux qu'il y ait cette diversité qui permet d'ouvrir notre champ poétique.

N.A. On a déjà reproché à la génération de l'Hexagone le thème du pays, le thème de la femme associée au pays par exemple.

F.O. Pour moi, ce fut souvent du sentimentalisme tout ça et il ne fallait pas que ça dure plus longtemps parce que c'était une impasse sur le plan de l'écriture.

N.A. L'ensemble de votre œuvre poétique est donc fondé sur un rapport personnel, je dirais en tant qu'homme, avec le monde.

F.O. C'est ça, beaucoup plus. II y a ainsi des poèmes qui sont marqués par des expériences vécues en Europe. J'essaie d'assimiler tout ce que je 
vis en somme. Cette question-là du Québec ne se pose pas pour moi sur le plan poétique.

N.A. Sauf pour Miron qui a réussi à en faire un thème...

F.O. Ç'a été sa vie à lui...

N.A. ... et une esthétique, c'est-à-dire qu'il a réussi à associer son identité personnelle à l'identité collective, à une langue...

F.O. Parce qu'il a vécu sa langue à travers sa propre vie qui est indissociable de notre lutte collective.

N.A. On ne s'invente pas de thèmes en poésie, on n'écrit que ce que l'on a vécu le plus pronfondément, n'est-ce pas?

F.O. Exactement!

N.A. C'est pourquoi un critique ne peut pas reprocher à un poète...

F.O. On ne peut reprocher à Mallarmé de ne pas être Rimbaud... Cela n'a pas de sens.

N.A. Dans votre dernier recueil intitulé $A$ découvert, on reconnaît Fernand Ouellette mais à peine. Les thèmes y sont à découvert, c'est-à-dire que vous dénoncez les généraux qui mènent le monde, les prédateurs, l'ordre. Même la langue est très différente me semble-t-il, de celle de vos trois premiers recueils. Le style est plus limpide, il y a une clarté dans les images. Qu'est-ce qui supporte ces changements?

F.O. A un moment donné, il y a eu une sorte de condensation qui s'est faite dans mes rapports avec le monde, avec l'histoire immédiate, et des poèmes ont surgi très simplement parce qu'ils survenaient après trente ans d'écriture. Cette simplicité, en même temps que cette intensité sans laquelle il n'y a pas de poèsie véritable, cet accord entre l'émotion, l'écriture et I'histoire, ça se réussit ou non. II ne doit y avoir aucune concession, aucune baisse de tension. Parce qu'autrement on fait de l'écriture engagée. Mais j'espère bien ne jamais faire d'écriture engagée.

N.A. C'est ce que j'allais vous demander. II me semble qu'il y a quand même un engagement qui n'était pas présent dans d'autres recueils.

F.O. A mon sens, il était présent dans Séquences de l'aile, dans Le Soleil sous la mort, dans lci, ailleurs, la lumière; certains poèmes auraient pu paraître dans $A$ découvert, même si l'écriture n'est pas tout à fait la même.

N.A. Je veux dire que ces poèmes étaient rares dans l'ensemble de votre œuvre, alors qu'il y a une ligne d'engagement qui traverse tout $A$ découvert et qui est nouvelle.

F.O. Mais ce qui est volontaire, c'est le livre, parce que les poèmes ont été écrits parmi d'autres. 
N.A. II s'agit donc simplement d'un regroupement, d'un choix de composition?

F.O. En effet. J'ai extrait les poèmes d'A découvert d'un groupe de cent vingt-cinq poèmes inédits. Les éditions Parallèles me donnaient la possibilité de faire un livre de poèmes, qui, à sa façon, poursuivait la même visée que le livre d'essais qui devait paraître chez $\mathrm{HMH}$ sous le titre Écrire en notre temps, lequel, en partie du moins, est une dénonciation du mal de notre époque. J'étais heureux d'avoir l'occasion de faire ce geste de dénonciation sur deux plans d'écriture à la fois.

N.A. Ces poèmes ont été écrits à quelle époque?

F.O. Dans les trois dernières années.

N.A. On peut donc conclure que depuis les trois dernières années, il y a peut-être eu un changement chez Fernand Ouellette.

F.O. Oui et non. II ne faut pas oublier que les poèmes d'A découvert doivent être situés parmi cent vingt-cinq poèmes. II y a une multiplicité de thèmes dans l'ensemble. Bien entendu, si je les avais publiès en les disséminant dans le tout, ils auraient passé beaucoup plus inaperçus. C'est là que la volontè de construction d'un livre apparaît. Tu vois, $A$ découvert constituera la dernière partie du second tome de mes poèmes à paraître sous le titre En la nuit, la mer. Même s'ils n'ont pas été écrits les derniers, dans mon plan, et c'est là une volonté très claire, ils paraîtront en dernier. Un livre ce n'est pas une suite chronologique de textes.

N.A. Bon d'accord! Et si je prends ce recueil comme objet, il me semble traduire de plus une sorte de désenchantement ou un pessimisme assez évident du poète déçu par différents événements.

F.O. En tout cas je suis très blessé par l'histoire actuelle. Je ne vois pas beaucoup d'espoir. C'est-à-dire que le rapport que j'ai avec le monde est tendu, et même pessimiste. Disons que je n'ai pas une vision progressiste de l'histoire dans le sens très précis où je croirais que l'Humanité, de toute façon, va régler ses problèmes. Je crois au contraire à l'accident, à la catastrophe, je passe par l'eschatologie, un peu comme Pierre Vadeboncœur dans son dernier livre Les Deux Royaumes.

N.A. Ces années-ci, c'est un peu déprimant au Québec!

F.O. Non seulement au Québec mais aussi dans l'ensemble du monde. Il y a un coup de barre à droite évident. La révolution en Iran, c'est la révolution la plus significative sur ce plan-là, parce qu'elle est aussi religieuse. Ce mouvement universel et mondial actuellement, vers la droite, j'ai essayé de le comprendre dans un texte publié dans Écrire en notre temps et qui s'intitule "La Mort du futur". 
N.A. II faut dire aussi qu'il y a une grande déception vis-à-vis des pays dits socialistes.

F.O. C'est sûr, il y a une déception vis-à-vis de ce qu'on a pu appeler la notion progressiste de l'histoire. Tout le monde aujourd'hui est échaudé, à moins de se jeter à corps perdu dans une idéologie militante.

N.A. Pour revenir à la poésie, vous avez écrit dans Les Actes retrouvés : "Ce n'est que pour les aveugles que le poète semble parfois s'éloigner de son peuple". Je suis d'accord et pas d'accord, en ce sens que pour moi le poète est au cœur du peuple parce qu'il parle de l'homme quotidien, de ce qui l'entoure, mais il en parle dans un autre langage qui n'est pas celui du peuple. Ils ont peut-être le même référent mais ils n'ont pas la même façon d'utiliser le langage et ils ne saisissent pas ces mêmes objets de la même façon. Ce qui faisait dire à Valéry d'ailleurs que la poésie est un "langage dans un langage". Je voulais vous demander si culturellement le poète et le peuple, entre guillemets, pourront jamais se comprendre?

F.O. Enfin, il faudrait savoir ce que signifie "le peuple»? Si c'est la collectivité, disons la collectivité québécoise, si c'est la masse, pas plus que les autres, elle ne peut savoir comme telle ce qu'est la poésie.

N.A. Pourtant...

F.O. Ça concerne des individus qui vont acheter un livre de poèmes et qui vont vivre l'aventure poétique...

N.A. Mais le langage courant utilise, c'est ce qu'on arrive à démontrer dans nos universités, les mêmes structures que la poésie finalement, alors d'où vient la difficulté de saisir?

F.O. Quand quelqu'un ne comprend pas un poème, qu'est-ce qu'il dit ? II dit toujours « je ne comprends pas " parce qu'il veut comprendre justement. On ne peut pas parler du "peuple" au sens large. II faut dire pourquoi des individus, par exemple un ouvrier qui essaie de lire Mallarmé, ne peut comprendre. Ce n'est pas possible non plus. II ne peut pas comprendre parce qu'il s'imagine d'abord qu'il va piger avec toute sa raison, et que ça prend beaucoup de culture pour comprendre quelque chose en poésie. Ce qui est vrai c'est qu'il a fallu faire un cheminement. Rien n'est entièrement donné.

N.A. Alors il cherche un sens ailleurs.

F.O. Il cherche un sens hors du texte qu'il devrait pourtant prendre littéralement. Ne serait-il pas plus simple de recevoir le poème comme on reçoit un tableau, une œuvre musicale? Le difficile pour le lecteur peut être la concentration d'écriture, les rapports déroutants. D'une certaine façon, il faut avoir fait un chemin en poésie. Pour nous tous c'est la même difficulté. Prends une œuvre poussée à l'extrême comme Finnegans Wake de Joyce. L'œuvre véritable ne se donne que lentement, organiquement. 
N.A. II faut apprendre le langage...

F.O. II faut s'ouvrir au langage, être patient, attentif. Ce qui ne signifie pas qu'on ne peut pas avoir sur-le-champ une première émotion devant l'œuvre. Dire que ce n'est pas de la culture populaire mais élitiste ne veut rien dire. C'est une façon d'inoculer la médiocrité. On abaisse ainsi la télévision, par exemple, sur un plan strict de consommation. On bloque les choses en disant: "Les gens ne comprendront rien à ça”.

N.A. Ce qui n'est pas prouvé...

F.O. Ce n'est pas prouvé du tout parce que les gens du peuple qui sont sincères avec eux-mêmes ont une admiration pour ce qui se fait. Ils ne sont pas pédants.

N.A. Je connais un psychologue qui ne comprend rien à une métaphore. Ce n'est donc pas autant qu'on le croit une question de classe.

F.O. Non, parce que ma grand-mère maternelle, qui était d'origine paysanne et n'avait aucune instruction (elle avait peut-être une deuxième année) constamment découpait dans les journaux des poèmes qu'elle collait dans un agenda. D'où venait tout cela? Pourquoi trouvait-elle ce langage-là beau? Pour arriver au poème, il faut déjà une sorte de grâce, une sorte de talent particulier.

N.A. On retrouve finalement la même chose chez les intellectuels. II y en a beaucoup qui détestent la poésie, c'est-à-dire...

F.O. ... qui ne comprennent rien à la poésie. J'ai connu des romanciers qui avaient un mépris profond pour la poésie, qui la considéraient comme une impuissance, comme une incapacité de développer un texte ou d'écrire de la prose.

N.A. Si la poésie n'est pas lisible par tous, qu'est-ce que vous pensez, en quelques mots, de la poésie qui se veut engagée?

F.O. Si elle se "veut"... elle est mauvaise.

N.A. Si elle se veut n'importe quoi d'ailleurs...

F.O. Oui, si elle se “veut» n'importe quoi, c'est foutu. Mais si la poésie est emportée par un moment historique qui s'exprime avec force, alors il doit la traverser avec une nécessité d'écriture qui n'accepte aucune concession. En somme, le poète ne doit jamais tenter de faire passer un message, une idéologie, ou d'adapter son écriture à un message. Ce qui ne signifie pas que le poète est sans idéologie...

N.A. Le thème doit être senti au point de devenir écriture.

F.O. Je le pense. Ce que l'on dit travaille l'écriture dans tel sens. Il y a certaines choses que l'on ne parvient pas à saisir. Alors l'écriture se concentre. Disons que la concentration des métaphores, par exemple, sera plus forte dans la mesure où l'on n'arrive pas à saisir un éclat du 
réel. Parfois l'écriture parvient à une certaine maturité, une certaine limpidité par le temps et le travail. Elle semble naturellement se dégager des métaphores. Mais il ne faut rien brusquer. Si l'on veut aller trop vite, c'est peut-être plus le vide que le silence qui émerge. Il peut y avoir alors pour le lecteur superficiel une erreur de perspective. Ce qui ne signifie pas que l'écriture à haute densité métaphorique n'a pas atteint son point d'aboutissement, d'achèvement. Ça peut signifier qu'elle se manifeste alors dans un corps à corps avec l'ange, avec l'innommable. Enfin, ça peut-être une question de tempérament. Ma poésie demeure concentrée, elle ne fait pas de concessions même lorsque l'on dit qu'elle est "engagée». Mais il existe aussi une écriture un peu putain, raccoleuse qui vise à séduire les gens sur le plan idéologique. Mais nous ne sommes plus en poésie. Le malentendu est radical.

N.A. D'ailleurs il faut le dire: la poésie engagée n'a de sens que lorsqu'elle est dans le vécu du poète...

F.O. Quand elle est une nécessité du vécu...

N.A. ... qu'elle obéit à une nécessité de se dire aussi...

F.O. ... d'un destin et d'un vécu, c'est-à-dire quand ça fait partie de notre vie comme l'arbre et les autres choses font partie de notre vie. Arrive un point de maturité où ça s'exprime. Sinon c'est volontaire, politique, idéologique. C'est un autre travail qui se fait à ce moment-là. La poésie dérape... Mais il y a aussi des écritures qui sont fondées sur la rhétorique et les bons sentiments...

\section{Vers le roman}

N.A. Si nous parlions maintenant de votre prose. J'aimerais vous demander ce qui manque à la poésie pour que vous ayez senti le besoin d'écrire en prose?

F.O. Ça dépend de quelle prose on parle, si on parle de l'essai ou du roman, car ce sont deux choses différentes évidemment. L'essai, c'est le besoin de s'expliquer - en poésie on ne s'explique pas -, le besoin de dire des choses qu'autrement on ne pourrait pas saisir: tout ce qui est de l'ordre de la pensée, de la réflexion. Ce champ nécessite évidemment la prose. La crise du couple par exemple, j'ai essayé de l'exprimer dans le roman, car c'est une chose qui échappe complètement au poème, qui n'a rien à voir avec la matière poétique... C'est un problème de durée, d'espace et de lutte, de temps. Quand j'ai commencé à écrire mon premier roman, je ne savais pas ce que j'écrivais du tout, je ne savais pas que j'écrivais un roman.

N.A. Vous parlez évidemment du Tu regardais intensément Geneviève?

F.O. De Geneviève comme de La Mort vive, ce fut le même processus. 
Disons au fond que c'est la nature de ce qu'on a à dire qui appelle la prose ou le poème, c'est tout. Et le poème vient quand on ne sait surtout pas ce qu'on va dire...

N.A. Mais...

F.O. Tandis que dans la prose on dit beaucoup de choses...

N.A. ... mais qui sont d'un autre ordre...

F.O. Oui... d'ailleurs on ne sait pas ce qu'on ne veut pas dire non plus, ce qu'on ne peut pas dire...

N.A. Je crois qu'on dit surtout le désir de vie dans un poème, tandis que dans la prose on essaie de s'expliquer ce désir.

F.O. Quant à l'essai, on est déjà sur un autre plan. L'essai arrive lorsque l'on tente de s'expliquer, de comprendre aussi des choses à travers l'écriture. Car je crois à la pensée qui pense à travers l'écriture. Tant que je n'ai pas écrit, je n'ai pas pensé.

N.A. Tant qu'on n'a pas parlé, on n'a pas pensé...

F.O. C'est pour moi la même chose, c'est pourquoi j'ai besoin de la prose aussi.

N.A. Oui, mais dans Journal dénoué, j'ai lu ceci : «Autant je semble incapable de concevoir un roman, autaut je suis tenté par l'essai ". Vous avez fait les deux et maintenant vous semblez intéressé de plus en plus par le roman. Alors si on parlait d'abord de Tu regardais intensément Geneviève. Vous avez été très apprécié pour ce roman et parfois vertement critiqué. On vous reproche, par exemple, d'être trop près du poème ou bien on dit que l'auteur manque de recul par rapport à ses personnages. Qu'en pensez-vous vous-même? (Entre parenthèses, je ne suis pas tout à fait d'accord avec l'un de mes collègues qui remet en question les sources de votre roman, sa motivation psychologique même au lieu d'analyser le langage et le texte).

F.O. Quand j'ai commencé ce roman-là, j'ai commencé à écrire tout simplement une chose qui après le premier jet m'est apparue comme un roman. L'intention romanesque y était évidente; la construction, l'équilibre que je donnais aux choses.

N.A. C'est une architecture.

F.O. Pour moi, c'est une architecture et un travail délibéré de construction.

N.A. Vous avez construit votre roman de cette façon? Parce qu'à la lecture, on a plutôt l'impression que c'est très continu.

F.O. Oui, évidemment.

N.A. Vous avez donc réussi à effacer les traces de la construction? 
F.O. Bien sûr, il y a une construction. La première partie est toujours restée cette première partie, c'est la matière viscérale, l'épaisseur biologique du personnage. Dans un ton qui est presque de l'humour noir. La deuxième partie, c'est l'inconscient, les rêves. Et la troisième partie, c'est la conscience, le rapport avec l'histoire. Puis on rentre directement dans le quotidien, dans cette journée. Le roman se concentre dans une seule journée, il ne s'y passe rien, au fond, il n'y a pas de péripéties comme dans un roman classique. Le personnage se lève le matin et se couche le soir. Mais ce qui se passe c'est un regard sur lui-même, sur ce qu'il est, ce sont des coups de sonde dans sa mémoire, des évocations de son rapport avec sa femme.

N.A. Vous avez certainement lu La Modification de Michel Butor.

F.O. Oui... je l'ai lue, après.

N.A. Quoi?

F.O. Je l'ai lue après, mais je savais que Butor avait écrit La Modification à la seconde personne. Je savais quel ton Butor employait mais je n'avais pas lu le roman. D'ailleurs, j'ai écrit ma première version à la troisième personne.

N.A. C'est vrai?

F.O. Ça ne fonctionnait pas, cette distance de la troisième personne.

N.A. Et vous avez transposé?

F.O. J'ai tout repensé mon livre avec mon oreille, au fond. Quand je l'ai transposé à la seconde personne du singulier, c'est-à-dire quand j'ai entendu le «tu", j'ai entendu mon livre.

N.A. Bien, mais pourquoi le narrateur s'adresse-t-il à lui-même à la deuxième personne?

F.O. Ce n'était pas plausible autrement. Ça ne pouvait pas être quelqu'un d'extérieur au personnage qui raconte cette histoire éperdue, puisqu'il se parlait à lui-même. A lui-même, mais déjà pris comme objet, considéré à distance. Cela m'a permis de dédoubler les "je ". Le "je " de la parole, et le «je» de l'écriture, de ce qui a déjà été écrit, puisque le narrateur cite des passages de son journal. Le livre n'a commencé à fonctionner que lorsque j'ai entendu: «Tu regardais intensément Geneviève». J'ai senti qu'il y avait là l'accord inconsciemment cherché. Et ce ton n'a aucun rapport avec celui de Butor, à la seconde personne du pluriel, froid et distant.

N.A. Oui, c'est même un "on" pratiquement... ça devient impersonnel.

F.O. Evidemment.

N.A. Parce que Fernand Ouellette avait besoin de se dire des choses? 
F.O. C'est-à-dire le narrateur, puisque c'est un narrateur qui parle, avait besoin de dire certaines choses à celui qu'il est. II s'agit pour le narrateur, d'une forme de récit autobiographique... à travers la structure de la narration. Voilà la vérité du texte. Maintenant qu'est-ce qui est autobiographique? Quand les gens commencent à se poser la question...

N.A. Par rapport à l'auteur?

F.O. Par rapport à l'auteur...

N.A. Cela ne m'intéresse pas beaucoup, c'est très secondaire.

F.O. Ce qui est autobiographique dans la tension entre narrateur et auteur, c'est une autre question. En quoi cette matière brute devrait intéresser la critique? Doit-on, dans un compte rendu bref, se jeter sur l'aspect dit critique "génétique"? N'est-ce pas prématuré? Non! Ce qui devrait concerner la critique c'est de savoir si le texte fonctionne ou non sur le plan littèraire. En un sens la forme que j'ai choisie (ou qui s'est imposée) est celle du récit autobiographique. Le narrateur se raconte sa vie. Mais il ne s'agit pas de mon autobiographie. Si j'avais voulu reprendre mon autobiographie, je l'aurais fait sans détour. J'ai tout de même publié le Journal dénoué. Mais bien entendu, sur le plan de la sensibilité, de la perception des choses, il y a des éléments qu'on peut retrouver dans le Journal dénoué. Il s'agit du même écrivain. J'ai sans doute les mêmes problèmes, les mêmes désirs. En m'engageant dans cette aventure qu'est un roman, je fais aussi de l'autoportrait. C'est une question de tempérament, de type d'écrivain. Mais j'insiste encore sur la dimension romanesque qui a résulté d'un travail de trois ans. On n'a pas le droit de dire que l'écrivain est innocent.

N.A. Le question d'autobiographie, à mon avis, n'a pas beaucoup de sens, car on peut avoir vécu pendant une fraction de seconde une émotion très intense, et en faire un roman - par exemple on peut parler du suicide sans s'être suicidé! Mais ça pose un problème pour beaucoup de lecteurs, cependant.

F.O. Oui, oui, bien sûr, parce que le ton est tellement direct et dur que les lecteurs ont l'impression que c'est purement une confession autobiographique. Mais c'est inévitable puisque mon livre a la forme d'une confession autobiographique. C'est là qu'est l'ambiguïté.

N.A. Oui, c'est la confidence sans détour.

F.O. Bien entendu.

N.A. II y a aussi une mise à nu, à cause de cette forme, qui a surpris beaucoup de gens. Je crois qu'on ne s'attendait pas à ça de vous, après vos poèmes. Car un poète ne donne presque jamais de références biographiques. Or il s'agit ici d'événements très précis racontés en style clair, parfois dans une langue blanche. Quoique personnellement je trouve que c'est assez poétique malgré tout. 
F.O. Pour moi c'est un roman de poète, sur certains plans...

N.A. On l'a qualifié de prose froide, un peu dure...

F.O. Je ne pense pas...

N.A. Non, j'ai vu aussi que le roman est bourré de métaphores et d'envols poétiques.

F.O. J'ai tout de même fui le roman dit "poétique", parce que j'ai ça en horreur, sauf exception. Mais mon livre reste sans doute un roman de poète. Un ami, poète italien, vient de m'écrire à ce sujet: "ce que je trouve d'extraordinaire c'est la multiforme souplesse, la légère et pourtant "dure" pertinence du langage aux situations. Enfin, la véritable lumière du langage". Mais j'aimerais revenir sur un problème auquel on n'a pas assez réfléchi : l'autoportrait en littérature. Ce genre n'a jamais été un problème en peinture. II y a une longue tradition. D'ailleurs nul n'aurait reproché à Van Gogh d'avoir peint sa chambre jaune, ou sa chaise, ou ses bottines. Je ne parle pas des visages... Mais je prends un autre exemple. Changall a peint Bella dans sa robe blanche, bas mauves, mais une Bella qui le porte lui, Chagall, sur les épaules. (II a d'ailleurs raison de lui fermer un œil de sa main). Quel autoportrait, ou double-portrait magnifique quand on sait ce que fut la passion de Chagall pour Bella. Quel sens prend la toile, quelle confession! Bien! Or mon roman c'est un autoportrait de cette nature, je ne m'en cache pas. Je pourrais dire en blaguant, qu'à la différence de Belle, Geneviève s'enlève et le narrateur tombe à terre. Je procède ici par analogie pour montrer que je ne fais pas autre chose que ce que Changall a fait en peinture. Sauf, bien sûr que deux cents pages, c'est une durée et non l'instant d'un espace. Ça me permet de fouiller plus profondément, d'atteindre une certaine crudité qui en a scandalisé plusieurs. On pourrait parler des portraits que Cézanne a faits de sa femme, etc. En peinture, je le répète, on appelle ça un portrait ou un autoportrait. Pourquoi, en littérature, ce serait inadmissible? Est-ce que ça ne peut pas être l'une des formes du roman?

N.A. Je ne vois pourquoi ce ne serait pas une forme de littérature possible.

F.O. C'est ce que j'essaie de dire. Pourquoi l'autoportrait au sens large seraitil interdit en littérature? Pourquoi m'accuser de faire de l'autobiographie quand je fais de l'autoportrait? II y a là toute une part de fiction, d'imaginaire, toute la part de l'écriture, des désirs, des fantasmes, et pourquoi je n'aurais pas le droit de faire ça? On n'a jamais prétendu que ces choses personnelles n'appartenaient pas à la peinture et que Chagall était vaniteux. Pourquoi ne pas utiliser cette matière en littérature?

N.A. A mon avis, tous les objets se valent et je ne vois pas pourquoi on en exclurait. 
F.O. Ce travers de la critique m'a paru, je dois l'avouer, comme une chose un peu primaire, et je n'ai pas pris ça très au sérieux même si cela a pu me blesser. Je me suis dit: On manque de perspective, on s'attaque maintenant à un genre, le roman, dont on ne sait pas du tout ce que sera son avenir. Bon! moi j'ai fait un livre, je ne sais pas que c'est un roman, tu comprends... Le second je sais mieux que c'est un roman parce qu'il paraît plus classique. C'est sûr qu'il y a une intentionalité romanesque dans tout ça, dans la façon de construire, d'amener les choses, de choisir tel moment. Toute la construction se fait par l'imaginaire. Qu'il y ait eu dans ma vie un choc avec ma femme ou non, c'est secondaire, ça n'a aucun intérêt pour les autres. Des gens m'écrivent pour me dire qu'ils s'identifient au personnage masculin ou féminin, non pas selon le sexe mais selon l'expérience vécue.

N.A. Etonnant !

F.O. D'autres m'écrivent: C'est ma vie... certains disent que c'est un livre de misogyne, alors que des femmes affirment : enfin un homme capable de comprendre les femmes! C'est aux hommes de ma génération que le livre fait le plus mal, beaucoup plus qu'aux femmes.

N.A. Parce qu'ils s'y reconnaissent?

F.O. Parce qu'ils se sentent jugés et doivent se remettre en question, c'est viscéral. Ils ont vécu ces problèmes-là, ils se voient, et s'ils essaient de se regarder honnêtement, ce n'est pas facile d'accepter certaines choses. Le livre est assez brutal sur ce plan-là.

N.A. Vous décrivez là, je crois, des comportements typiques très répandus, non seulement dans votre génération...

F.O. Dans d'autres générations bien sûr, dans d'autres pays et d'autres cultures aussi.

N.A. Ce qui m'a étonné, c'est d'entendre Geneviève parler avec un fort accent québécois, marqué par des apostrophes.

F.O. On m'a déjà dit: «Tu n'aurais jamais dû faire parler Geneviève de cette façon-là »! Mais c'est un problème stylistique, parce qu'elle, elle n'écrit pas, elle parle, et c'est un cri de douleur car elle ne peut que hurler.

N.A. C'est-à-dire que vous avez voulu la montrer... à vif.

F.O. A vif... J'ai un petit chapitre là-dessus dans Écrire en notre temps, où j'ai essayé d'expliquer cette « rupture". Parce que la douleur est telle... j'ai essayé plusieurs autres solutions et ça ne fonctionnait pas.

N.A. II y a des phrases qui ne se disent pas sous l'effet de l'émotion, et il serait ridicule de faire disserter un personnage, d'accord.

F.O. Ça ne fonctionnait pas. Ça n'a fonctionné à mon oreille que lorsque le personnage s'est mis à hurler dans un style parlé qui a paru vulgaire à quelques-uns de mes amis. Certains me disent que l'écrivain s'est 
donné le beau rôle: il écrit bien. D'autres qu'il est impitoyable envers lui-même. Ce qui est vrai c'est que le personnage-écrivain, dans le livre, ne parle pas, il écrit. La seulè foís où il parle, c'est pour répondre à Geneviève, et alors il parle comme elle. Il n'y a donc pas de discrimination. Il parle parce qu'il est moins en colère, à ce moment-là, qu'en douleur. II se contrôle. Tandis que Geneviève réagit comme un volcan. La lave s'accumule depuis vingt ans. Elle hurle de douleur.

N.A. Oui? Ça ne m'a pas frappé pourtant, de sa part à lui.

F.O. Ça frappe moins parce que la réplique est très brève. Tandis que l'éruption de Geneviève est littéralement un cri de douleur. Son "cri" m'a posé des problèmes. Je ne pouvais pas le rapporter en style indirect, sans l'affaiblir considérablement. Je l'ai donc épinglé comme une photographie réaliste dans un tableau. C'est une faille véritable. Et ce n'est qu'à la toute fin, dans la dernière des cinq versions, que j'ai trouvé ma solution.

N.A. Et l'écriture romanesque, c'est une expérience que vous avez faite à mesure en y prenant plaisir?

F.O. Bien sûr, c'est très agréable. Ce qui est très agréable c'est de vivre longtemps avec un texte, contrairement à l'expérience du poème.

N.A. Je comprends très bien.

F.O. C'est un plaisir presque sensuel de l'écriture qui dure longtemps...

N.A. N'est pas là la difficulté de la prose d'ailleurs? Le fait d'être une architecture, quelque chose qui s'inscrit dans la durée, alors que le poème est instantané. La prose peut vous obséder pendant des jours, des mois...

F.O. Un livre comme Geneviève ou la Mort vive, c'est une expérience d'écriture qui dure trois ans et plus. Mais le premier jet fut écrit respectivement en quinze et treize jours, à un an d'intervalle. Je reçois l'écriture alors comme une précipitation...

N.A. D'abord un premier jet rapide!

\section{F.O. D'accord!}

N.A. Vous retravaillez le texte avec des procédures différentes mais un peu comme vous travaillez le poème?

F.O. C'est-à-dire que je suis forcément obligé de revenir sur le texte après. Disons que pour mon second roman la construction n'a jamais changé.

N.A. La structure générale demeure?

F.O. Le texte est là, la matière est là mais ce n'est pas encore achevé comme écriture. Il peut y avoir encore des problèmes de construction, de chute. 
N.A. De son côté, Hubert Aquin écrivait avec des plans bien précis, structurés.

F.O. C'est tout le contraire de moi effectivement. Ce sont peut-être les "véritables romanciers" qui ne peuvent écrire un roman qu'avec un plan. Moi j'en suis incapable.

N.A. "Véritables» romanciers?

F.O. Chacun de nous réagit selon son tempérament. Pour moi, ça ne se passe pas autrement qu'en poésie, c'est-à-dire que c'est une précipitation psychique qui, au lieu de durer quinze vers dure deux cent cinquante pages ou treize jours. C'est exténuant évidemment, parce que j'écris jusqu'à dix heures par jour. Je suis incapable de me ralentir, je suis emporté par le texte et l'histoire et il faut que je me rende au bout. Cette «méthode» a des inconvénients.

N.A. Tu regardais intensément Geneviève date donc de quelques années?

F.O. De 1975 .

N.A. II y a un aspect qui m'intrigue dans votre premier roman: comment conciliez-vous cette langue, quand même très ouvragée, les références musicales et culturelles surtout, avec le parti pris de réalisme et de confidence?

F.O. C'est un jeu de construction et d'éclairages. Ce travail me permet de transmuter le ton ou de le moduler. Par exemple, toutes ces introductions poétiques qui semblent n'avoir aucun rapport avec le texte qui va suivre sont des failles, bien entendu, mais elles sont surtout des musiques qui me permettent de donner la tonalité, des éclairages, à des niveaux différents.

N.A. II y a des rapports symboliques entre les tableaux et le récit des événements?

F.O. Certainement. Le tableau de Corot que j'évoque, par exemple, avant de parler du grand-père. J'y vois un rapport de ton, d'ambiance, de lumière. Ailleurs, lorsque je décris un tableau de Vermeer, c'est une façon d'établir un rapport d'atmosphère lumineuse entre la pièce qui sera décrite, et dont la dominante sera la lumière, une certaine lumière, et le tableau de Vermeer. Le rapport s'établit donc à la fois sur un plan émotif et sur un plan poétique. Je veux signifier que c'est principalement la lumière de la pièce des personnages qui importe. Une sorte d'atmosphère satinée.

N.A. Pour percevoir cela, il faut tout de même une lecture très attentive du texte.

F.O. A mon sens, il n'a pas encore été lu, ce texte. J'allais dire que la critique est toujours rapide par la force des choses.

N.A. A la parution d'un livre oui, il faut ensuite attendre une étude... 
F.O. Joseph Bonenfant a commençé à parler un peu de la forme, mais on n'a pas encore parlé, à mon sens, de ce qu'est la forme du livre, ni de son travail..

N.A. Enfin on y viendra. Passons, si vous voulez, au plan thématique. Si Fernand Ouellette est toujours un peu idéaliste, est-ce que cela ne transparaît pas dans les réponses que le narrateur apporte à ses propres questions, c'est-à-dire des réponses qui tendent à maintenir l'amour comme éternel et absolu malgré l'expérience quotidienne. Autrement dit, est-ce que vous ne tentez pas, est-ce que le narrateur ne tente pas de maintenir l'amour au niveau du mythe dans ce roman?

F.O. Je ne sais pas s'il tente de le maintenir au niveau de mythe. Je me suis demandé s'il ne s'efforçait pas de le saisir dans cet instant où l'amour ne semble pas pouvoir mourir, mais aspire à durer, alors qu'il est soumis à une tension quotidienne qui le rend en fait très vulnérable. Peut-être est-ce une façon, à travers l'écriture, de le voir et de lui donner une sorte d'éternité. Je ne sais pas. Mais ce n'est certainement pas un a priori idéologique où je dirais que par définition l'amour est éternel. Pas du tout!

N.A. D'accord, mais l'impression qui se dégage est que vous souhaiteriez que l'amour soit absolu.

F.O. Ce problème est reposé dans mon deuxième roman où le personnage féminin dit au personnage principal (et elle n'a pas de lien amoureux avec lui): Tu sais très bien, toi comme moi, comme Pierre, que le mariage ne peut pas être absolu et que ce serait une erreur de le penser, car on sait très bien qu'on est mortel. Mais il se passe autre chose chez le narrateur de Geneviève. Rien n'est vraiment vécu sur un plan idéólogique mais tout sur un plan viscéral. II croit que son amour devrait durer parce qu'il aime tout simplement. Ainsi il est peut-être porté à l'idéaliser, à lui donner une lumière qu'il n'a sans doute pas. Des lecteurs diront que ce couple-là va se séparer, d'autres que les personnages vont continuer de vivre ensemble, ou encore que les personnages n'ont rien de commun. Mais il est sûr que le narrateur, lui, qui raconte le moment de la crise de son couple, croit aimer et par conséquent voudrait que son couple dure. Il lui donne donc par son écriture, son regard de poète, une certaine dimension mythique.

N.A. Je faisais allusion à la conception même de l'amour. En ce sens que, avec ce que je connaissais du thème de l'amour dans votre poésie, où l'amour est mythifié positivement, c'est-à-dire est pris comme absolu peut-être le seul absolu ou la seule force capable de tranformer les êtres...

F.O. Je crois que c'est la seule façon que nous ayons. Si nous n'avions pas l'amour, au sens large, nos autres puissances s'effriteraient. Que nous resterait-il? Est-ce que je mythifie l'amour? J'hésite à employer ce vocabulaire, parce que pour moi c'est une donnée vitale, primordiale. 
N.A. Il est certain, en tout cas, que dans Tu regardais intensément Geneviève, il n'y a pas finalement le constat brutal des faits purs et simples, et il y a toute une argumentation sous-jacente qui tente de justifier, de comprendre, et d'essayer de faire durer à tout prix ce sentiment.

F.O. Oui, mais comprendre et justifier non pas in abstracto mais par rapport à lui-même, pour que Geneviève comprenne. Chez lui, c'est l'instinct de défense qui parle pour rétablir un meilleur rapport de compréhension. Je pense que le narrateur pourrait se tromper sur son propre amour, penser qu'il aime sans aimer vraiment et faire tout cet effort disons d'introspection pour se persuader lui-même qu'il aime.

N.A. Oui, mais la conclusion n'est pas dégagée dans ce sens-là ?

F.O. Non, je l'ai laissée ouverte complètement...

N.A. Et c'est pour ça qu'on peut comprendre de façon contradictoire, en faire un livre anti-féministe de surexploitation du moi masculin, ou au contraire une remise en question, une réévaluation des comportements masculins?

F.O. Bien sûr, je ne vois pas du tout ce livre comme anti-féministe. Je ne dis pas que le personnage n'a pas été misogyne, je pense qu'il l'a été et c'est pour cela qu'il y a eu crise dans le couple, il a été du moins aveugle et inconscient comme beaucoup d'hommes, sinon misogyne.

N.A. Nous parlons toujours du narrateur?

F.O. Oui, du narrateur comme personnage. Au fond, c'est le roman de la crise et non pas celui de l'histoire du couple. On m'a reproché de ne pas montrer tous les éléments de tendresse qu'il y avait dans ce couple, leur passé... leur vie commune quoi! Mais ce n'est pas ça, le livre, c'est celui de la crise.

N.A. Même là, je considère qu'il y a de la tendresse.

F.O. II y en a, c'est évident. Les gens qui disent qu'il n'y a pas de tendresse, c'est qu'ils n'ont pas su lire le livre. Ils me disent: "Le narrateur écrit à la fin "si je vivais la tendresse". Mais c'est parce qu'il la vit déjà qu'il peut la proposer, même comme un désir non accompli. Car la tendresse n'est jamais acquise. II faut toujours y tendre.

N.A. Le narrateur ne lui a pas donné la parole, à elle.

F.O. Elle ne l'a pas prise. Elle a donc sa part de responsabilité. Un couple implique un dialogue. Quand Geneviève prend la parole, c'est dans un acte de violence morale. Finalement le narrateur prend conscience de ses erreurs passées, de son comportement, et il se dit qu'il doit maintenant se taire et entourer Geneviève de tendresse. C'est ça le fond du livre. Il est sûr qu'à la fin, le lecteur fait un choix, car il se projette luimême dans le couple. Je citerais Proust qui écrivait: "chaque lecteur est, quand il lit, le propre lecteur de soi-même». Je pense donc que les 
lecteurs se lisent eux-mêmes. Le critique se dévoile. II l'oublie trop facilement. C'est pourquoi certaines critiques, par leur ton, m'ont fait sourire.

N.A. N'est-ce pas tout ce qu'un lecteur peut faire de toute façon?

F.O. Bien sûr. Alors les problèmes que certaines personnes voient dans mon roman, peuvent être leurs problèmes de lecteur.

\section{Un deuxième roman: La Mort vive}

N.A. Maintenant, que nous réserve La Mort vive? Vous avez commencé d'en parler, tout à l'heure, est-ce du même genre?

F.O. Pas du tout! II ne s'agit pas du même thème. Geneviève est une histoire d'amour. La Mort vive se concentre sur le phénomène de “création ». Un peintre se cherche à travers son œuvre et semble aboutir à l'impasse. Contrairement à Geneviève, plusieurs personnages gravitent autour du personnage principal. Mais les tensions dramatiques surviennent tout de même dans ses rapports avec les femmes. II n'y a pas vraiment de désaccord avec ses amis qui sont comme lui, créateurs, mais plutôt une profonde amitié, une complicité.

N.A. L'action se passe où, exactement?

F.O. A Montréal, ici, et en dehors de Montréal, s'étendant sur une période d'environ treize ou quatorze ans. C'est toute une autre facture que le premier, et il est écrit à la troisième personne. II y a cependant beaucoup d'éléments du "Je".

N.A. Que le narrateur reprend?

F.O. Oui, que le narrateur prend chez le personnage principal surtout. C'est un narrateur omniscient. En fait, le travail a porté autant sur le plan de la narration, sur l'équilibre des voix, que sur celui de l'exploration réflexive par le personnage principal. Non seulement l'impasse semble totale dans ses rapports avec les femmes, mais surtout sur le plan du rapport de son art avec sa société, son temps. Tout se passe comme si sa vie était un échec.

N.A. Alors, il y a plus d'épaisseur que dans Tu regardais intensément Geneviève?

F.O. Comme projet, oui. Et aussi parce qu'il y a la notion de temps qui n'est pas la même. La Mort vive couvre la période de 61 à 72 ou 73 . J'ai dû faire attention pour ne pas me laisser entrainer par les événements politiques, parce que je pouvais détruire mon livre très facilement.

N.A. Les éléments politiques?

F.O. Par exemple, j'avais une longue discussion sur les événements d'octobre. Or je me suis rendu compte qu'à ce moment précis du roman 
cette discussion n'entrait pas dans la logique de mon personnage. $\mathrm{Ce}$ qui importait plus que tout, c'était le choc qu'il venait d'avoir d'une femme. Il était trop obsédé par elle, alors, pour s'intéresser vraiment à la discussion de ses amis.

N.A. Cela aurait eu une incidence sur les rapports des personnages entre eux.

F.O. Ca aurait été une digression malheureuse pour la tension de mon récit. Tout à l'heure, on parlait d'engagement. On est souvent porté à introduire une matière pour se situer volontairement dans une époque...

N.A. Encore faut-il y aller d'une façon naturelle et non pas volontaire ou artificielle...

F.O. Oui! Car on ne peut rien faire de volontaire sans se casser la figure. II faut qu'il y ait une cohérence constante. Comme dans Geneviève, d'ailleurs, je ne sais pas si mon roman a une fin. La fin apparente est plus tragique évidemment que dans Geneviève. On pourrait croire qu'il s'agit d'un suicide. Ça peut sembler évident pour certains lecteurs.

N.A. Evident, dans quel sens?

F.O. Pour le lecteur ça peut être un suicide parce que le personnage disparaît dans des conditions mystérieuses. Mais on n'est pas tout à fait sûr qu'il soit mort. Tout dépend de la manière qu'on vit avec le personnage au cours des trois cents pages. L'idée que j'en ai, c'est qu'il s'agit d'une réussite spirituelle. II va au bout de sa quête, même si sa vie semble un échec sur le plan concret. II meurt peut-être, mais ça pourrait être un accident lié à sa faiblesse cardiaque. Mais je n'en suis pas sûr, il pourrait s'agir également d'une forme de suicide qui viendrait à la fois de son déséquilibre et de sa plénitude. II semble halluciné. II comparaît devant les "Régentes", personnages inhumains d'un tableau de Frans Hals. Tout est donc possible.

N.A. Est-ce qu'on ne pensera pas au suicide à cause des échecs?

F.O. C'est-à-dire que tout est échec... On dit que c'est un livre sombre parce qu'évidemment, sur le plan de la peinture, ses vernissages, ses expositions, sa possibilité d'être exposé à New York, à Paris, tout ça c'est un échec complet. Sa peinture a atteint un niveau d'expression que trop peu de gens peuvent percevoir, au fond. C'est un peu comme s'il atteignait une lumière que peu de gens peuvent voir, parce que peu de gens ont fait un chemin spirituel qui est équivalent au sien. On pourrait donc penser au suicide.

N.A. Est-ce qu'il n'y a pas un peu du Québécois là-dessous? De l'artiste québécois ou même de l'écrivain québécois dans son milieu...

F.O. Sans doute, mais je pense que c'est universel aussi. J'aurais pu faire le même roman si le personnage avait vécu à Paris ou à New York. 
N.A. Oui, mais au Québec, il y a quelque chose de particulier, le pays est petit...

F.O. Ce qui aggrave tout...

N.A. Et l'on se retrouve sans support psychologique, non seulement économique mais psychologique...

F.O. Sans véritable support culturel aussi, parce que l'un des facteurs de la réussite d'un peintre est sa situation socio-historique. Au XIV ${ }^{\ominus}$ siècle, il fallait venir à Paris; à la Renaissance, à Rome Florence. Au XIX siècle et au début du $X X^{\ominus}$ siècle, à Paris. II n'y avait pas de réussite mondiale possible sans passer par ces lieux. C'était donc une condition liée à des hasards historiques pour le peintre. Par exemple, au XV siècle, la peinture s'est déplacée de Paris en Italie. Ce qui s'est fait alors en Provence, n'a pas eu le rayonnement et l'influence qui auraient été possibles si ce n'avait été que de la seule qualité plastique. C'est donc ce support culturel, socio-historique que nous n'avons pas comme Québécois, et nous ne sommes pas les seuls. Aujourd'hui, il faut aller à Paris, comme Riopelle, ou à New York, franchir ces Himalayas dira mon peintre-personnage. Hors de ces villes, qui sont des carrefours d'influences, le peintre ou l'écrivain ont peu de chances d'irradier. Quelques prix Nobel...

N.A. Mais nous sommes en dehors de ces deux influences!

F.O. Exactement! C'est pour ça que la vie d'écrivain québécois est si difficile: on ne peut pas être perçu en ligne directe par les autres cultures, on n'existe pas...

N.A. C'est un problème très grave d'ailleurs... pour la motivation.

F.O. C'est très grave parce que pour être perçu, il faut être à Paris, il faut être prix Goncourt.

N.A. Et encore! Dans le cas d'Antonine Maillet, on a récompensé le folklore, ce qui ne correspond pas à notre lecture du texte.

F.O. Je sais bien. C'est là tout l'ambiguïté de nos rapports avec la France. Le personnage de mon peintre vit cette impasse. Sa peinture, c'est une réussite mais seulement quelques amis le savent. Lui-même ne le sait pas parce qu'il dèsespère d'atteindre ses objectifs. II sent qu'il est rendu à son plus haut point d'expression parce qu'il ne peut pas aller plus loin, il le sait dans la mesure où il cesse de peindre un peu comme Rimbaud cesse d'écrire et il s'en va alors à Povungnituk, où il disparaît. C'est la lumière qui lui fait choisir le Nord.

N.A. C'est une belle disparition...

F.O. II disparaît tout simplement, dans la neige, comme s'il avait voulu entrer physiquement dans son dernier tableau, celui d'une alliance vive de la neige et de la lumière. Un peu comme ce peintre d'un conte chinois qui, 
n'acceptant pas le reproche que lui fait l'empereur, se retourne et entre dans son tableau pour y disparaître. Mon peintre aussi disparaît dans le dernier tableau de son désir, physiquement.

N.A. En tout cas il a dit: C'est mon dernier tableau...

F.O. Il a dit si ça pouvait être... mon dernier tableau. Mais je laisse le livre ouvert.

\section{Fernand Ouellette essayiste}

N.A. Abordons brièvement le dernier versant de Fernand Ouellette, celui de l'essayiste. Pourquoi vous êtes-vous tellement intéressé à l'essai ? Estce que vous vous intéressez à tout, comme Valéry? Est-ce pour parler de Fernand Ouellette qui reconnaît ses parentés spirituelles, je songe à Varèse pour la musique, à Kierkegaard et l'angoisse, à Cendrars dont vous dites ce que vous pourriez dire de vous-même: "Son être est précisément paradoxal parce qu'il est vivant». Pourquoi ces essais?

F.O. Disons d'abord que plusieurs études ont été provoquées par les événements, par la situation. Je pense à “la Lutte des langues» et à quelques autres articles que j’ai faits dans les années 1963-1964. Mais ce n'est pas dans ma nature. Je préfère maintenant écrire des choses très librement, sans apparat. C'était du travail d'intellectuel. Je ne le méprise pas, mais ce n'est pas dans mes cordes. L'essai me tentera toujours. C'est une façon de penser, une façon de me saisir et de capter ce qui m'entoure. Comme au fond je vis assez seul, que je parle peu avec les autres, c'est un moyen pour moi d'arriver au dialogue. J'ai des conversations avec des amis, bien sûr, mais ce n'est pas toujours là qu'on met au point ce qu'on voudrait dire. C'est à travers l'écriture qu'on arrive à cerner des choses qu'on s'explique mal ou qu'on perçoit mal. Et comme je ne suis pas dans l'enseignement, où l'on est obligé d'une façon presque continue de formuler les choses, l'essai est pour moi une façon, comme solitaire, d'arriver à parler aux autres.

N.A. C'est la société dans laquelle vous vivez qui vous intéresse et que vous essayez de comprendre?

F.O. Oui, j'essaie de comprendre ce que je suis moi-même, comme homme, puis les problèmes qui agitent la société. Et parfois mon point de vue semblait assez technique mais...

N.A. Ce qui frappe effectivement dans vos essais, c'est le côté scientifique. Quand vous parlez de la langue par exemple, vous avez des références linguistiques précises en plus des réflexions personnelles. II ne s'agit pas d'un travail spontané.

F.O. Non, bien sûr. Mais je considère ça comme des accidents de parcours, c'est-à-dire comme des choses que j'aurai dû faire à cause des circons- 
tances. A cause de ma collaboration à la revue "Liberté », de l'obligation de travailler à des questions précises.

N.A. Mais des questions qui vous passionnaient?

F.O. Forcément! Mais disons que c'était des choses quand même plus accidentelles. Je voudrais aller librement vers ce qui vraiment me passionne et non plus vers des choses qui me torturent. Je travaille actuellement à un livre qui comporterait à la fois des poèmes, et des essais sur la peinture, etc. Quant à ma relation, disons tendue et douloureuse avec l'époque, l'histoire, je pense l'avoir exprimée dans un des derniers livres, en particulier dans Ecrire en notre temps, et d'une autre façon dans mes poèmes.

N.A. Vous tendez plus à présent vers le littéraire?

F.O. Oui, des textes qui vont vers d'autres questions au fond. II faut quand même continuer à vivre, autrement aussi bien se taire tout de suite, car si je prends l'histoire comme absolu, l'histoire va m'étouffer, il n'y a pas d'issue, et je ne pourrais plus écrire. Si je veux continuer, il faut que je prenne d'autres directions! Ça peut être la peinture ou d'autres choses.

N.A. En critique littéraire, vous avez des réticences devant une "science de la littérature". Vous parliez même de la sémiologie, déjả, en 1970 et vous écriviez: "Bien que nécessaire, cette connaissance a ses propres limites et n'exclut pas l'intuition que le poète peut avoir d'un texte». Je crois que vous n'êtes pas le seul à le penser. A mon avis, si la première impression intuitive d'un texte est fausse, la "science" démontrera n'importe quoi, sur tout ce qu'elle veut, et cela repose justement le problème de l'épaisseur du texte, de son obscurité matérielle et des sens nombreux qu'il y a toujours à une ouvre. Est-ce que l'auteur est nécessairement toujours meilleur juge de sa propre œuvre?

F.O. II n'est pas nécessairement le meilleur juge. Pour ma part, j'accepte toutes les formes de critique, que ce soit une critique sémiologique, thématique, structuraliste, etc. Je ne suis pas anti-universitaire et certainement pas anti-intelligence. Je suis au contraire entièrement d'accord avec toutes les formes de travail de l'intelligence et je m'élève toujours contre les écrivains qui se moquent de ce travail, parce que je pense que nous, on ne peut pas le faire tout en écrivant. Mais aucune de ces lectures-là n'est satisfaisante parce qu'elle n'est pas, comme le travail d'écrivain lui-même, une lecture totalisante. Et le rêve de tout critique c'est d'atteindre une critique totalisante.

N.A. Mais on en est loin!

F.O. On en est loin et si on arrivait à une science vaste et totalisante, ce serait extraordinaire, ce serait un peu l'utopie du critique.

N.A. Non, parce qu'il y a beaucoup de choses qui échappent au critique, les accidents d'écriture, les contingences. Tout à l'heure nous parlions de 
l'arbre, il y a aussi le téléphone, n'importe quel événement qui provoque et influence l'écriture. Quel critique pourrait remonter à toutes les sources de l'inspiration, ou aux déterminations de l'écriture?

F.O. II suffit d'accepter la limite d'une chose. Je suis entièrement d'accord avec des gens qui travaillent sur un plan précis sans prétendre donner une réponse définitive à l'œuuvre.

N.A. Donc à la condition qu'ils situent leur méthode?

F.O. A condition qu'ils situent leur niveau de lecture. Alors je sais ou crois savoir de quoi ils parlent. Enfin, il peut arriver que je sois moins intéressé par tel type de lecture. Auerbach, Curtius, pour le moment, me satisfont davantage. J'aime la critique dans la tradition des romanistes allemands qui ont une vue synthétique, nourrie de multiples cultures. Sur le plan d'une "poétique», on est en pleine tentative d'une science littéraire. C'est tout un autre domaine. Mais c'est un travail de l'intelligence, de la culture, qui est en soi intéressant et sans doute nécessaire, si une science littéraire devait exister un jour.

N.A. On éclaire moins l'œuvre que la science?

F.O. ... que les phénomènes des genres, des catégories... Je refuse donc qu'on méprise ce travail de l'intelligence, mais ça ne signifie pas qu'il me satisfait, surtout si on prétend tout réduire à une forme de critique. Par exemple, établir une équation entre le réel et l'écriture, au moment où les hypothèses socialistes prétendaient que la littérature est le reflet du réel. On tombe épisodiquement dans ce piège.

N.A. La vieille théorie du reflet...

F.O. On sait aujourd'hui que même les socialistes, même un Barberis, n'acceptent plus du tout cette théorie qui conduisait à une impasse.

N.A. Pour revenir à une question qui nous préoccupe à l'Université, je crois toujours que l'intuition perçoit mieux une œuvre que n'importe quel montage théorique qui fait l'économie d'une intuition de base. Les grilles peuvent cŏnduire à une mauvaise lecture sans cette intuition.

F.O. II s'agit de l'intuition d'un point de vue particulier toujours, et d'une situation dans l'espace-temps.

N.A. Mais si l'on n'a pas saisi l'ensemble d'une œuvre, comment peut-on parler d'un aspect particulier?

F.O. Bien sûr! André Belleau disait une chose très simple là-dessus, c'est qu'au fond un critique a beau avoir la science littéraire, s'il n'a pas d'oreille, il ne sera jamais capable de lire une œuvre. Parce que pour lire Proust, il faut avoir de l'oreille et combien de gens font de la critique sans avoir de l'oreille, sans savoir ce qu'est la phrase et l'écriture. Ils n'ont pas d'oreille, alors ils essaient de "comprendre"... faute de pouvoir entendre. 
N.A. Eux aussi cherchent autre chose?

F.O. Autre chose, Ils diront "autres choses"...

N.A. Est-ce que vous trouvez qu'on a bien lu vos œuvres, dans l'ensemble, c'est-à-dire est-ce que vos intentions d'écrivain ont été bien perçues?

F.O. Pour la poésie en général, ça commence. Mais il m'est difficile de juger parce que je n'ai pas fait ce travail-là sur ma propre poésie ; je connais le travail critique de l'écriture évidemment, mais c'est autre chose.

N.A. Ce n'est pas le même recul.

F.O. Le recul critique, je ne l'ai pas, et quand je lis Pierre Nepveu, ii m'apporte des choses, il m'ouvre des points de vue que je n'avais pas vus. Je crois beaucoup au lecteur, je crois que les lectures que les gens font vont ouvrir le sens de mes textes.

N.A. A moins que cette lecture aille complètement en sens contraire...

F.O. Alors, à ce moment-là, il y a une rupture, et je n'ai pas de lien du tout avec ces lectures-là, si la tangente est telle que ça n'a plus de rapport avec ce que, moi, je pense avoir mis dans le texte. C'est normal. Et je peux dire qu'il y a plusieurs aspects dans mes textes qui ne sont pas encore éclairés. Toute cette sensibilité dont je parlais tout à l'heure et que j'appellerais l'irradiation, la vibration lumineuse du texte, pour moi c'est primordial...

N.A. Cette irradiation est liée aux sonorités?

F.O. Aux sonorités et à ce qu'on voit visuellement. C'est le travail du regard dans le texte du poème. Par exemple quand Jouve dit: "II fait beau sur cette pierre à mourir de ciel bleu ", je vois tout l'éclatement de la lumière en même temps que le sens. Ce travail du regard, la façon de voir les choses, c'est ça la lumière. Pour moi le regard, loreille, le son, la lumière, sont indissociables.

N.A. Tout cela est-il lié aussi à la nature de la métaphore chez vous?

F.O. A la nature de la métaphore, beaucoup au regard, à la vision unique du poète.

N.A. Oui, mais vous employez le mot «regard" à la fois au sens premier et d'une façon métaphorique, et je sens qu'il y a quelque chose d'autre làdedans...

F.O. Je dis souvent au fond que le véritable regard du poète, c'est le regard qui tente de saisir ce qu'il ne peut pas voir justement. Si la poésie pour moi consistait seulement à rendre compte de ce que je vois, elle n'aurait plus d'intérêt puisqu'ayant vu, je n'ai pas besoin de faire l'expérience de l'écriture pour ça...

N.A. La poésie exprime ce qui ne peut pas se dire, l'indicible? 
F.O. Ce qu'on dit l'indicible, ce qui ne peut pas être vu, ce qu'on tente de cerner.

N.A. C'est la définition que je donnerais de la poésie.

F.O. Oui, c'est bien la visée de la poésie, parce que justement c'est ce qu'on ne peut pas dire que l'on tente de saisir, et qu'on ne pourra jamais dire, c'est pourquoi on continue d'écrire. Je parle de ce problème dans Écrire en notre temps, où j'essaie de saisir ce qu'est la poésie dans un travail plus réflexif. Disons que j'ai une certaine vision de la poésie, c'est tout.

N.A. Mais pour essayer d'en comprendre le fonctionnement?

F.O. Essayer de comprendre ce qui se passe. Ma réflexion sur la poésie bouge beaucoup et c'est pourquoi je me méfie d'une " théorie" poétique, parce qu'elle serait figée...

N.A. Mais vous essayez même de saisir une démarche au fur et à mesure qu'elle se produit?

F.O. Oui. Je me rends compte par exemple qu'aujourd'hui, douze ans après, je ne récrirais pas "Le Poème et le Poétique" de la même façon ni dans les mêmes termes. Je tente de poursuivre une réflexion qui est parallèle à ma démarche. Mais je ne considère pas du tout que c'est de la théorie! On tente de prendre une distance, on se remet en question constamment et on semble se contredire souvent d'ailleurs. C'est inévitable parce qu'on n'est plus sensible aux mêmes mots, aux mêmes nuances. Ce mot-là, choisi il y a dix ans, ne nous intéresse plus beaucoup, on sent que ce n'est jamais tout à fait ça...

N.A. Certains conçoivent l'écriture comme un acte douloureux. Est-ce que vous définiriez l'acte d'écrire dans ces termes-là?

F.O. Douloureux, oui et non, parce que c'est en même temps...

N.A. ... un plaisir?

F.O. Un plaisir et une joie aussi. Si ce n'était pas déjà un plaisir, on ne pourrait pas le faire, cela n'aurait pas de señs. Dans $A$ découvert, ce sont des poèmes très douloureux et si j'arrive à parler quand même de ces choses, c'est que le plaisir, à un moment donné, l'emporte sur la douleur, autrement il n'y aurait pas d'écriture.

N.A. Surtout le plaisir de fabriquer le poème?

F.O. De faire quelque chose...

N.A. Le plaisir esthétique finalement?

F.O. Celui de faire un objet, oui le plaisir de faire l'objet l'emporte et c'est vrai dans tous les arts de toutes les époques. Même pour Homère qui disait que son effroi le paralysait en évoquant telle scène. 
N.A. La prose aurait un avantage sur la poésie du point de vue du plaisir d'écrire?

F.O. Oui et non. C'est-à-dire que le plaisir est plus soutenu, on se bat avec un texte qui a beaucoup plus d'épaisseur et les difficultés ne sont pas du tout du même ordre: les difficultés de construction, de syntaxe, de correspondance des temps, etc. Le poète, en général, n'a aucune conscience de cette "cuisine" parce que ses problèmes ne se posent pas sur ce plan-là, il est presque hors du temps dans son écriture.

N.A. Le poème est une intuition immédiate...

F.O. C'est comme un tableau, c'est une chose saisie, presque cristallisée. Il y a d'ailleurs un rapport très intéressant entre la peinture et la poésie, parce qu'on saisit le tableau d'un coup pour ensuite rentrer dedans, le regarder, comme on fait avec un poème, on y rentre après un premier choc global. Tandis que le roman, c'est un autre travail, et en même temps peut-être plus douloureux parce que l'on se bat beaucoup plus, le travail matériel est considérable quand il faut le reprendre. J'ai repris des pages peut-être quinze fois, vingt fois, pour mon premier roman, j'ai dû écrire dans les deux milles feuillets.

N.A. Qui sont tous des choix possibles?

F.O. Toutes ces feuilles écrites, pour essayer d'arriver à saisir la chose un peu mieux. La seule dactylographie du manuscrit, c'est assez épuisant ! Pour le poème, on ne connaît pas ces «ennuis". Je pense que ce sont deux mondes.

N.A. Deux arts complètement différents?

F.O. Un critique m'a dit que si je n'avais pas fait ce travail de prose, je n'aurais pas pu écrire les poèmes d'A découvert, et il voit l'émergence de ces poèmes comme une sorte de résultante de mon travail en prose.

N.A. C'est une hypothèse très intéressante. Je vous poserais une dernjère question, si vous le permettez. Qu'est-ce que ça signifie pour vous, d'écrire au Québec en 1980 ?

F.O. Bon! Y continuer à écrire tout simplement! En 1980, pour moi, le problème ne se pose pas comme pour certains écrivains.

N.A. Si la question nationale...

F.O. Si la question nationale ne se règle pas, ça ne change pas mon écriture parce que celle-ci n'est pas liée à la question nationale.

N.A. II y a tout de même une motivation...

F.O. C'est sûr que je vais avoir un choc. Le danger serait de désespérer en disant: Qu'est-ce que je fais là? Quel intérêt y a-t-il à continuer d'écrire? En ce qui me concerne, je pense que je vais passer à travers cette épreuve-là, si jamais elle se produisait, parce que toute mon 
écriture depuis trente ans n'est pas soutenue par cette obsession. Elle en souffre, mais elle n'est pas liée à cette question. Je pourrais donc m'en sortir mieux que celui dont toute la pensée serait liée au problème de l'indépendance, qui lui aurait donné sa vie.

N.A. N'y a-t-il pas une sorte de désengagement des écrivains québécois en général, depuis 1976 ?

F.O. II y a une cette expérience que des écrivains ont vécue en Algèrie: ils étaient tellement liés à la guerre de l'Indépendance, que toute l'attention de leur écriture était prise par l'histoire. Quand l'Indépendance s'est produite, ils ne pouvaient plus écrire.

N.A. Ils n'avaient plus rien à dire?

F.O. Le sujet était brûlé même si ça s'est terminé d'une façon positive puisqu'ils ont accédé à l'Indépendance. Pour nous...

N.A. Ce serait l'inverse, en cas d'échec.

F.O. Ce serait l'inverse, mais on pourrait toujours avoir l'espoir d'y arriver un jour. Et en même temps on pourrait désespérer d'écrire pour un peuple qui ne veut même pas s'affirmer comme tel.

N.A. N'est-ce pas la plus forte tentation, du fait aussi qu'on est très peu nombreux!

F.O. Oui, et puis, on écrit aussi en français...

N.A. Oui, en français mais...

F.O. C'est une double difficulté. On envie tous un peu les Danois, qui ont une petite littérature nationale, d'accord, mais aussi une langue qui est la leur. Ils ne sont pas obligés de passer par Paris pour écrire en danois, alors que pour nous, à Paris, et ailleurs dans le monde, on ne sait pas toujours que le Québec existe, qu'il y a des écrivains de langue française.

N.A. On le sait de plus en plus, et il est consolant de penser qu'un jour ou l'autre on écrira peut-être pour l'ensemble des francophones?

F.O. Je l'espère! Parce qu'au fond tout le travail des Rencontres québécoises internationales des écrivains, c'est pour arriver à faire prendre conscience à nombre d'écrivains dans le monde que la littérature québécoise existe et le Québec aussi. Enfin c'est une des causes à laquelle j'ai travaillé pendant huit ans. Car mon travail en écriture est aussi indissociable de ma fonction sociale comme écrivain. 